\title{
Molecular detection of spotted fever group rickettsiae in ticks parasitizing pet dogs in Shihezi City, northwestern China
}

\author{
Wurelihazi Hazihan ${ }^{1} \cdot$ Zhihui Dong $^{2}$ - Liping Guo ${ }^{3}$. Kadyken Rizabek ${ }^{4}$. \\ Dzhunysov Askar ${ }^{4}$. Kulmanova Gulzhan ${ }^{4}$. Mahanov Kudaibergen ${ }^{4}$. \\ Akishev Nurlan Kenjebaevich ${ }^{4} \cdot$ Tolegen Talgat $^{4} \cdot$ Kenesbay Kairullayev $^{4}$. \\ Yuanzhi Wang ${ }^{2}$
}

Received: 18 July 2018 / Accepted: 28 December 2018 / Published online: 16 January 2019

(c) The Author(s) 2019

\begin{abstract}
A total of 178 adult ticks were collected from 32 pet dogs from five veterinary clinics in Shihezi City, Xinjiang Uygur Autonomous Region (XUAR), northwestern China. All the ticks were identified by comprehensive morphological and genetic analyses, and rickettsiae were detected by seven Rickettsia-specific genetic markers in the ticks. The ticks collected were identified as Rhipicephalus sanguineus sensu lato. Twenty-one of the 178 samples (11.8\%) were positive for rickettsiae. Among these, in 13 (61.9\%) samples Candidatus R. barbariae were identified, in five (23.8\%) samples $R$. massiliae, and in three (14.3\%) samples $R$. conorii. This study indicates that more attention should be paid to rickettsial infection in pet dogs and their ticks, because the latter may pose an epidemiological risk for tick-borne transmission of rickettsiae to human beings.
\end{abstract}

Keywords Rhipicephalus sanguineus sensu lato $\cdot$ Spotted fever group rickettsiae $\cdot$ Pet dogs $\cdot$ Northwestern China

\section{Introduction}

Ticks are among the most common ectoparasites of dogs, also involved in the transmission of a number of major diseases in both dogs and humans (Chomel 2011; Dantas-Torres and Otranto 2016). Tick-borne rickettsioses are caused by the spotted fever group rickettsiae (SFGR) of the genus Rickettsia, which contains approximately 20 species, and many of which are established or emerging human pathogens (Wood et al. 2012). Besides, more and

Wurelihazi Hazihan, Zhihui Dong and Liping Guo are equal contributors.

Electronic supplementary material The online version of this article (https://doi.org/10.1007/s1049 3-018-00337-1) contains supplementary material, which is available to authorized users.

Yuanzhi Wang

wangyuanzhi621@126.com

Extended author information available on the last page of the article 
more new SFGR species have been found across the world, as a result of range expansion of tick populations, changes in landscape and climate, and more accurate diagnostic testing (Trotta et al. 2012; Yunik et al. 2015).

Due to the emerging and re-emerging nature of tick-borne diseases in humans, increasing focus has been placed on research of ticks parasitizing domestic animals (Hiraoka et al. 2005). As in many other countries, in China the dog has become a bonded family member. Regardless the benefits of having pet dogs, pathogens carried by ticks are potentially transmissible to humans, which may represent a health risk, especially to children, elderly people and immunocompromised individuals (Dantas-Torres and Otranto 2014). To date, at least three protozoan (Theileria, Babesia and Hepatozoon) and five bacterial (Anaplasma, Ehrlichia, Rickettsia, Coxiella and Bartonella) tick-borne genera have been reported in domestic dogs around the globe (Beck et al. 2009; Brown et al. 2006; Buhariwalla et al. 1996; Camacho et al. 2001; Conrad et al. 1991; Kaewkong et al. 2014; Kamani et al. 2013; Levin et al.2012; Mokhtar et al. 2013; Yabsley et al. 2008). In Jiangxi Province, mid-eastern China, Babesia canis vogeli and Babesia gibsoni were molecularly detected in 780 dog ticks (749 Rhipicephalus sanguineus, 16 Haemaphysalis campaulata and 15 Haemaphysalis verticalis), while all sampled dog ticks were negative for rickettsial agents (Zheng et al. 2017). In Xinjiang Uygur Autonomous Region (XUAR), northwestern China, rickettsial agents were prevalent in ticks infesting both domestic animals and wildlife (Guo et al. 2015, 2016). However, there is limited knowledge on the species of ticks infesting dogs. Here a molecular investigation was carried out for rickettsial agents in pet dog ticks.

\section{Materials and methods}

\section{Collection and identification of ticks}

In 2016-2017, ticks were sampled from 32 pet dogs presented at five veterinary clinics with symptoms of depression, weight loss and anorexia in Shihezi City ( $483 \mathrm{~m}$ above sea level, at $44^{\circ} 268129^{\prime} \mathrm{N} 86^{\circ} 0627148^{\prime} \mathrm{E}$ ), the northwestern China. The ticks were placed in tubes with $75 \%$ ethanol and stored at $-80{ }^{\circ} \mathrm{C}$. All of the ticks were identified morphologically according to previous reports (Filippova 1997; Dantas-Torres et al. 2013a, b). Twenty-nine representative ticks, with 4-6 ticks at each veterinary clinic, were used to analyze tick species and genetic diversity based on partial mitochondrial $16 S$ rRNA (460 bp), $12 S$ rRNA (400 bp) and coxI (889 bp) gene sequences (Szabó et al. 2005; Chen et al. 2014).

\section{DNA extraction and molecular detection}

After detailed morphological analysis, genomic DNA was extracted from each individual tick using the TIANamp Genomic DNA Kit (TianGen, Beijing, China). The ticks were mechanically crushed twice in sterile water for $15 \mathrm{~min}$ and then dried on sterile paper, suspended in $200 \mu \mathrm{l}$ tissue lysis buffer and $40 \mu \mathrm{l}$ proteinase $\mathrm{K}(100 \mu \mathrm{g} / \mathrm{ml})$, and incubated overnight at $56{ }^{\circ} \mathrm{C}$. The final elution volume was $60 \mu \mathrm{l}$. Subsequently, the polymerase chain reaction (PCR) technology was used to detect rickettsial agents with seven genetic markers for DNA fragments [434-, 1332-, 1060-, 488-, 920-, 491-, and 812-bp products of the genes encoding the 17 kilodalton antigen $(17-k D a), 16 \mathrm{~S}$ rRNA( $(r r s)$, citrate synthase $(\mathrm{gltA})$, surface cell antigen 1 (scal), PS120-protein-encoding gene (gene D), and outer membrane proteins A and B (ompA and ompB)] (Anstead and Chilton 2013; Chilton 2013; Sekeyova 
et al. 2001; Wei et al. 2015). (Table 1). Rickettsia aeschlimannii from Rh. turanicus and double-distilled water were used, respectively, as positive and negative controls (Wei et al. 2015). The PCR products were purified using the TIANgel Midi Purification Kit (TIANGEN, Beijing, China), and then subjected to sequencing (BGI, Shenzhen, China). Phylogenetic analyses were conducted used MEGA version 6.0 based on the $17 \mathrm{kDa}$-rrsgltA-ompA-ompB-gene $D$ concatenated sequence data of the rickettsiae by Maximum Likelihood (ML) and Neighbor-Joining (NJ) methods (Tamura et al. 2013).

\section{Results}

A total of 178 adult ticks (76 males and 102 females) were collected and morphologically identified as Rh. sanguineus sensu lato. (Fig. 1). The sequencing data from the 29 representative ticks confirmed the morphological results based on Basic Local Alignment Search Tool (BLAST) analyses of $16 S$ rRNA, $12 S$ rRNA and coxl. Rhipicephalus sanguineus s.l. in this study had $93.3-93.8 \%$ pairwise nucleotide sequence identity to genome sequences of the reference strains Rh. sanguineus (GenBank: JX416325) for three genes analyzed. Our data were deposited in the GenBank database (16SrRNA: KY069269, 12S rRNA: KY069270, and coxl: KY069271).

Twenty-one of the 178 samples $(11.8 \%)$ were positive for SFG rickettsiae. Of which, thirteen (61.9\%) were identified as Candidatus R. barbariae, five $(23.8 \%)$ as $R$. massiliae, and three (14.3\%) as $R$. conorii subsp. indica. (Additional Table 2; Fig. 2). Rickettsia massiliae and $R$. conorii subsp. indica had $99.8-100 \%$ and $99.3-100 \%$ pairwise nucleotide sequence identities to the corresponding sequences of the reference strains $R$. massiliae MTU5 (GenBank: CP000683) and R. conorii str. Malish 7 (GenBank: AE006914) for seven genetic markers, respectively. Candidatus R. barbariae in dog ticks showed $100 \%$ pairwise nucleotide sequence identity to the corresponding sequences of Candidatus R. barbariae in the flea Vermipsylla alakurt (according to the seven genetic markers, in GenBank: KT284715, KU645283, KT284716, KU645284, KT284717, KT284718, KU645286, respectively). Detailed similarities of the sequences in this study are shown in Additional Table 1. All the sequences of Rickettsia spp. obtained in this study were deposited in GenBank [17 kDa: KY069262-KY069264; rrs: KY069266-KY069268; gltA: KY069259-KY069261; scal: KY069254-KY069255, KY069265; ompA: KY069256-KY069258; ompB: KY069248-KY069250; gene D: KY069251-KY069253].

\section{Discussion}

In the present study, ticks collected from pet dogs were used to identify rickettsial agents in Shihezi City, northwestern China. Candidatus R. barbariae, $R$. conorii subsp. indica and $R$. massiliae were molecularly detected. Importantly, these rickettsial agents were shown to be present both in pet dog ticks (reported here) and in sheep ticks (Guo et al 2016), which data raise both veterinary and public health concerns in northwestern China.

Candidatus R. barbariae was originally reported from Rhipicephalus bursa ticks in Portugal (de Sousa et al. 2006), and later confirmed and characterized by five genetic markers (gltA, ompA, ompB, sca4 and rrs) from Rh. turanicus in Italy (Mura et al. 2008). Subsequently, Candidatus R. barbariae was also detected in Rh. turanicus from Cyprus and in Rh. sanguineus from Israel (Chochlakis et al. 2012; Waner et al. 2014). In 2016, our 


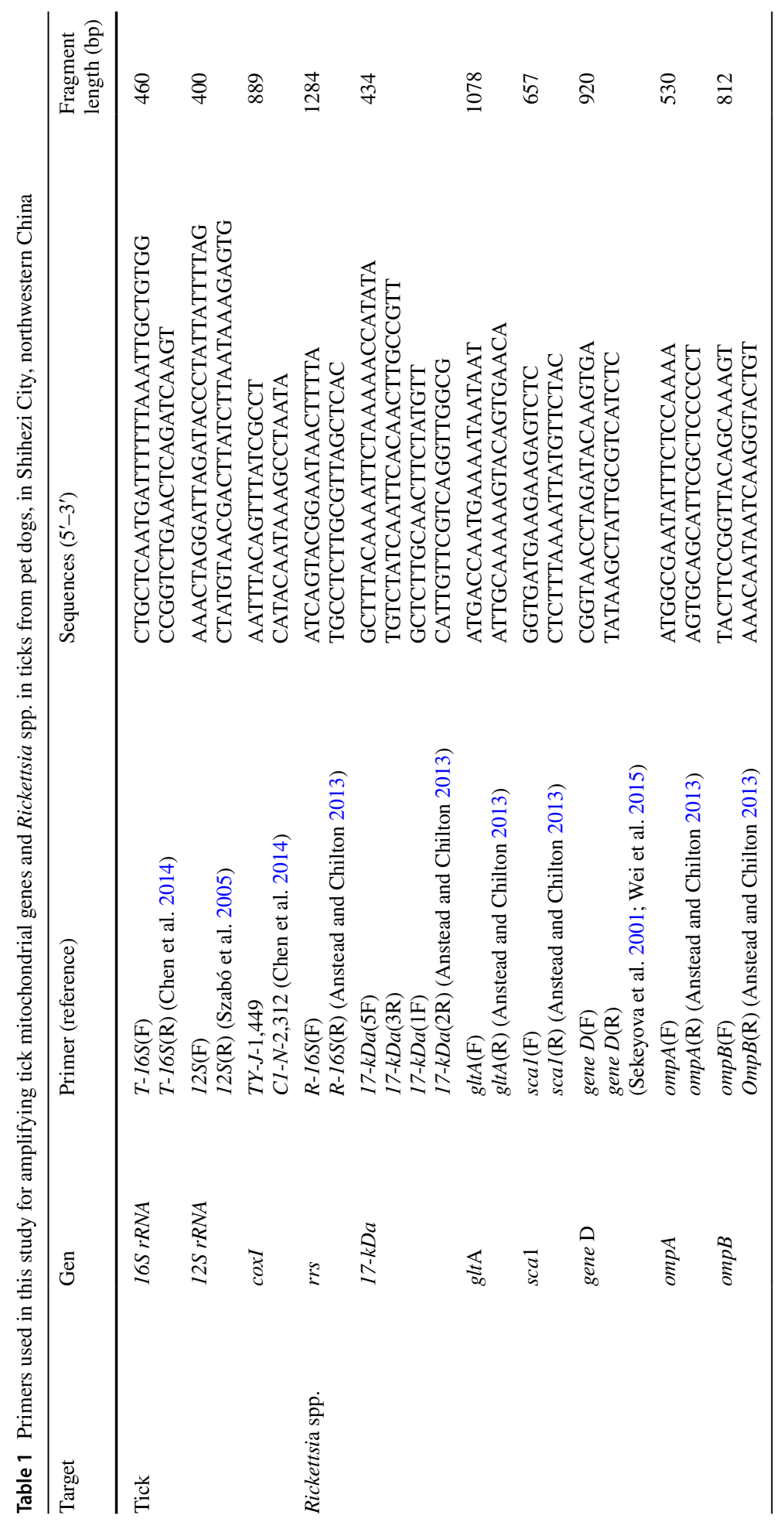



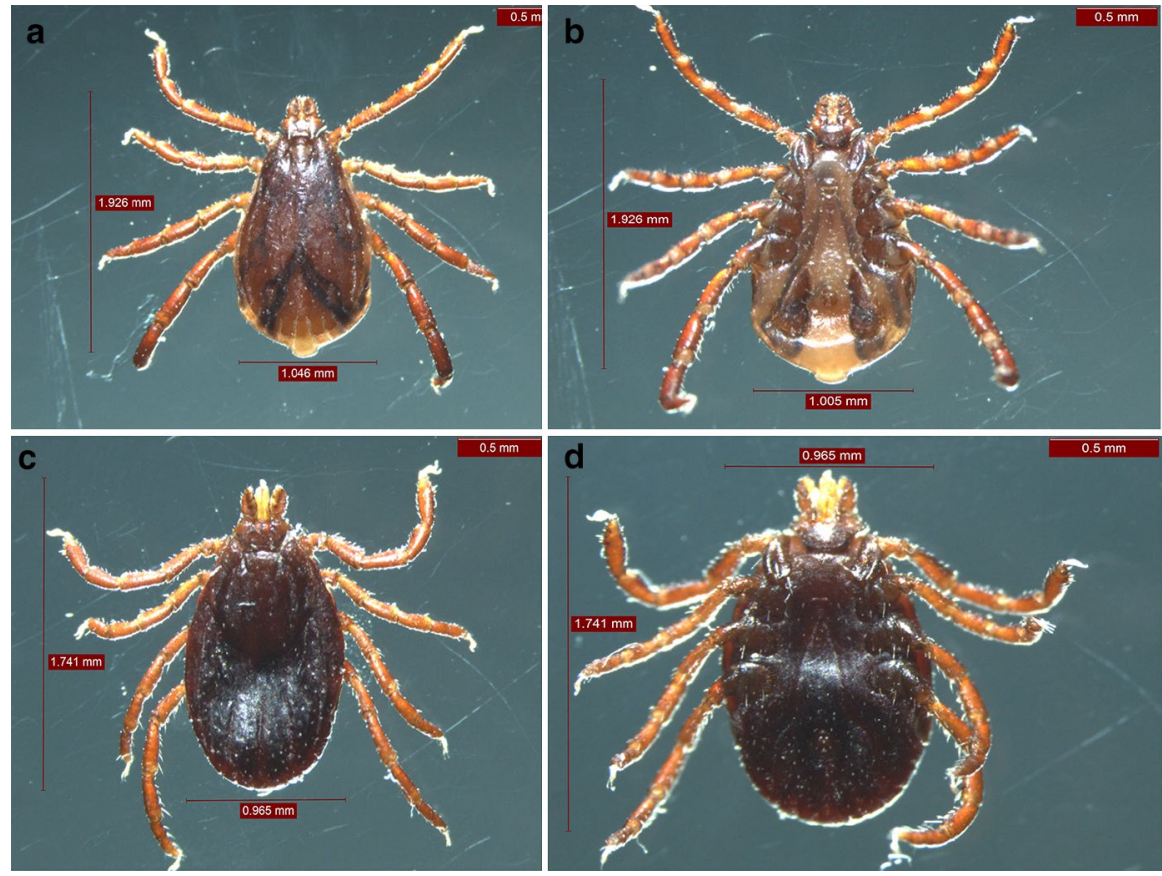

Fig. 1 Morphological analysis of Rhipicephalus sanguineus sensu lato collected from pet dogs. a Male, dorsal; b male, ventral; c female, dorsal; d female, ventral

investigation revealed that Candidatus R. barbariae is present in Vermipsylla alakurt fleas and Rh. turanicus ticks from grazing sheep (Guo et al. 2016; Zhao et al. 2016). Here, molecular evidence of Candidatus R. barbariae is provided in pet dog ticks (Rh. sanguineus s.1.).

The other two Rickettsia species, $R$. conorii subsp. indica and $R$. massiliae, had lower rates of positivity $[1.7 \%(3 / 178)$ and $2.8 \%(5 / 178)$, respectively] compared to the data from grazing sheep (Wei et al. 2015; Guo et al. 2016), which might be explained by differences in tick numbers per host, as well as by varying susceptibility to rickettsiae among host species. To the best of our knowledge, however, the clinical cases were caused by $R$. conorii subsp. indica and $R$. massiliae (Cavagnaro et al. 2008; Vitale et al. 2006). Although there is no documented clinical case of rickettsia infection from pet dog ticks in China to date, more measures should be carried out to prevent its risk to dog owners, taking into account the synanthropic nature of $R h$. sanguineus s.l. A diversity of tick-borne pathogens, including Anaplasma, Babesia, Borrelia, Ehrlichia and Theileriai spp. has recently been molecularly detected in Russia (Livanova et al. 2018). This, together with the present findings, draw the attention to not-yet known risks associated with tick-borne rickettsiae in several regions of Asia. 


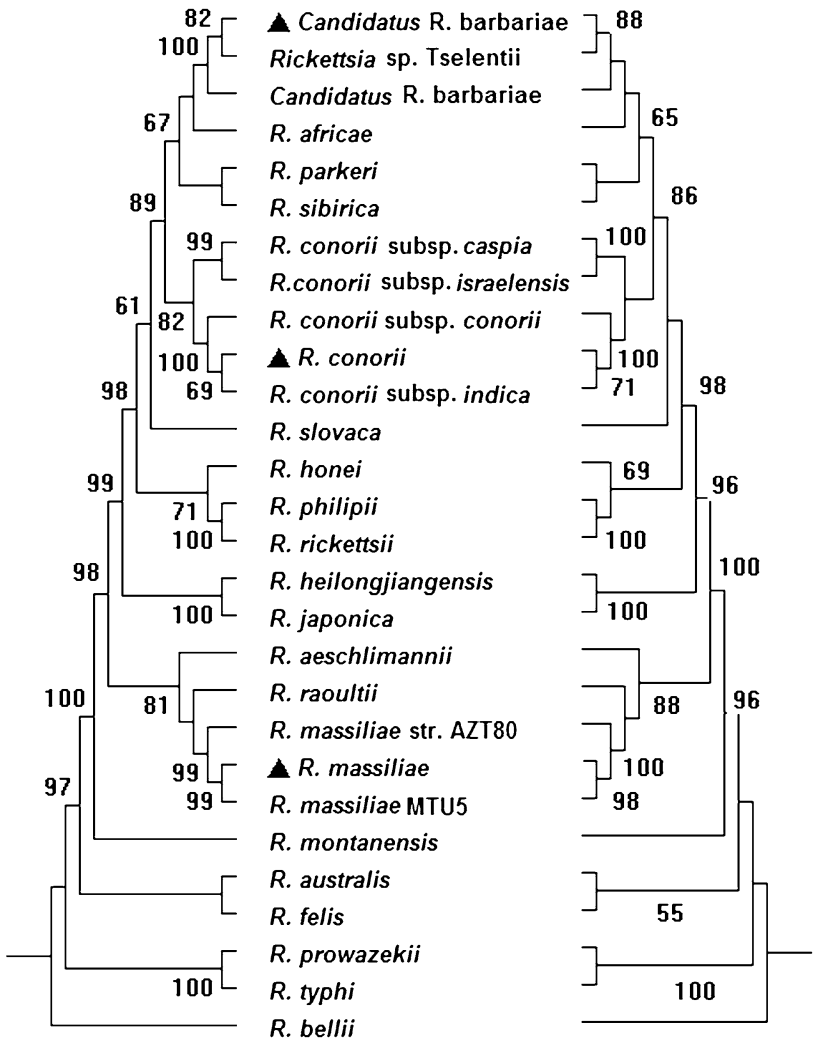

Fig. 2 Phylogenetic relationships of Rickettsia spp. inferred from 17 kDa-rrs-gltA-ompA-ompB-gene D using the Maximum-Likelihood method (left) and Neighbor-Joining method (right). The bootstrap consensus tree inferred from 1000 replicates and bootstrap replicates with value less than 50\% were collapsed. Phylogenetic analyses were conducted in MEGA6.0. Rickettsiae obtained in this study were marked as " $\boldsymbol{\Delta}$ ", and sequences for rickettsia species retrieved from the GenBank database, Rickettsia bellii was used as the outgroup (see Additional Table 2). The scale bar represents the inferred substitutions per nucleotide site

\section{Conclusions}

Three SFGR members, the $R$. conorii subsp. indica, Candidatus R. barbariae and $R$. massiliae, were molecularly detected in Rh. sanguineus s.l. ticks from pet dogs in Shihezi City, northwestern China. The study expands the range of tick-borne pathogens in pet dog ticks in Central Asia. Effective measures should be taken into consideration to prevent tickborne transmission of rickettsiae to human beings.

Acknowledgements We would like to thank Pro. Renfu Shao, Xingquan Zhu and Sándor Hornok revising the manuscript.

Author contributions YZW conceived and designed the study. LPG and KR critically revised the manuscript. HZ and ZHD analyzed the data and drafted the manuscript. DA and KG conducted the morphological test of dog ticks. MK, ANK, TT and KK conducted molecular analyses. All authors read and approved the final manuscript. 
Funding This research was supported in part by the National Key Research \& Development Program of China (2018ZX10101002-002-007), the National Natural Science Foundation of China (Grant Nos. 81560338 and U1503283), and the International Cooperation Projects of Xinjiang BINGTUAN (No. 2016AH001).

\section{Compliance with ethical standards}

Conflict of interest The authors declare that they have no competing interests.

Ethical approval This study was approved by the Animal Ethics Committee of Shihezi University (Approval No. AECSU2015-22).

Informed consent Informed consent was obtained from all the owners.

Availability of data and material The datasets supporting the conclusions of this article are included within the article and the newly-generated sequences were deposited in the GenBank database.

Open Access This article is distributed under the terms of the Creative Commons Attribution 4.0 International License (http://creativecommons.org/licenses/by/4.0/), which permits unrestricted use, distribution, and reproduction in any medium, provided you give appropriate credit to the original author(s) and the source, provide a link to the Creative Commons license, and indicate if changes were made.

\section{References}

Anstead CA, Chilton NB (2013) A novel Rickettsia species detected in vole ticks (Ixodes angustus) from Western Canada. Appl Environ Microbiol 79(24):7583-7589

Beck R, Vojta L, Mrljak V, Marinculić A, Beck A, Živičnjak T, Cacciò SM (2009) Diversity of Babesia and Theileria species in symptomatic and asymptomatic dogs in Croatia. Int J Parasitol 39(7):843-848

Brown GK, Canfield PJ, Dunstan RH, Roberts TK, Martin AR, Brown CS, Irving R (2006) Detection of Anaplasma platys and Babesia canis vogeli and their impact on platelet numbers in free-roaming dogs associated with remote Aboriginal communities in Australia. Aust Vet J 84(9):321-325

Buhariwalla F, Cann B, Marrie TJ (1996) A Dog-Related Outbreak of Q Fever. Clin Infect Dis 23(4):753-755

Camacho AT, Pallas E, Gestal JJ, Guitián FJ, Olmeda AS, Goethert HK, Telford SR (2001) Infection of dogs in north-west Spain with a Babesia microti-like agent. Vet Rec 149:552-555

Cavagnaro CS, Brady KA, Siegel C (2008) Fever After International Travel. Clin Pediatr Emerg Med 9(4):250-257

Chen Z, Li Y, Ren Q, Luo J, Liu Z, Zhou X, Liu G, Luo J, Luo J, Yin Het (2014) Dermacentor everestianus Hirst, 1926 (Acari: Ixodidae): phylogenetic status inferred from molecular characteristics. Parasitol Res 113(10):3773-3779

Chilton CAANB (2013) A novel rickettsia species detected in vole ticks (Ixodes angustus) from western Canada. Appl Environ Microbiol 79(24):7583-7589

Chochlakis D, Ioannou I, Sandalakis V, Dimitriou T, Kassinis N, Papadopoulos B, Tselentis Y, Psaroulaki A (2012) Spotted fever group rickettsiae in ticks in cyprus. Microb Ecol 63(2):314-323

Chomel B (2011) Tick-borne infections in dogs_-An emerging infectious threat. Vet Parasitol 179:294-301

Conrad P, Thomford J, Yamane I, Whiting J, Bosma L, Uno T, Holshuh HJ, Shelly S (1991) Hemolytic anemia caused by Babesia gibsoni infection in dogs. J Am Vet Med Assoc 199:601-605

Dantas-Torres F, Otranto D (2014) Dogs, cats, parasites, and humans in Brazil: opening the black box. Parasit Vectors 7:22

Dantas-Torres F, Otranto D (2016) Best practices for preventing vector-borne diseases in dogs and humans. Trends Parasitol 31(1):43-55

Dantas-Torres F, Latrofa MS, Annoscia G, Giannelli A, Parisi A, Otranto D (2013a) Morphological and genetic diversity of Rhipicephalus sanguineus sensu lato from the new and old worlds. Parasit Vectors 6(1):213-213

Dantas-Torres F, Capelli G, Giannelli A, Ramos RAN, Lia RP, Cantacessi C, de Caperariis D, De Tommasi AS, Latrofa MS, Lacasella V, et al (2013b) Efficacy of an imidacloprid/flumethrin collar against fleas, ticks and tick-borne pathogens in dogs. Parasite Vectors 6(1):245-245 
de Sousa R, Barata C, Vitorino L, Santos-Silva M, Carrapato C, Torgal J, Walker D, Bacellar F (2006) Rickettsia sibirica isolation from a patient and detection in ticks, Portugal. Emerg Infect Dis 12(7):1103-1108

Filippova NA (1997) Fauna of russia and neighbouring countries. Ixodid ticks of subfamily amblyomminae. Nauka Publishing House, Moscow (ISBN: 5-02-026081-9)

Guo LP, Mu LM, Xu J, Jiang SH, Wang AD, Chen CF, Guo G, Zhang WJ, Wang YZ (2015) Rickettsia raoultii in Haemaphysalis erinacei from marbled polecats, China-Kazakhstan border. Parasit Vectors 8:461. https://doi.org/10.1186/s13071-015-1065-1

Guo LP, Jiang SH, Liu D, Wang sw, Chen CF, Wang YZ (2016) Emerging spotted fever group rickettsiae in Ticks, northwestern China. Ticks Tick Borne Dis 7(6):1146-1150

Hiraoka H, Shimada Y, Sakata Y, Watanabe M, Itamoto K, Okuda M, Inokuma H (2005) Detection of rickettsial DNA in ixodid ticks recovered from dogs and cats in Japan. J Vet Med Sci 67(12):1217-1222

Kaewkong W, Intapan PM, Sanpool O, Janwan P, Thanchomnang T, Kongklieng A, Tantrawatpan C, Boonmars T, Lulitanond V, Taweethavonsawat P, Chungpivat S, Maleewong W (2014) High throughput pyrosequencing technology for molecular differential detection of Babesia vogeli. Hepatozoon canis, Ehrlichia canis and Anaplasma platys in canine blood samples. Ticks Tick Borne Dis 5:381-385

Kamani J, Baneth G, Mumcuoglu KY, Waziri NE, Eyal O, Guthmann Y, Harrus S (2013) Molecular detection and characterization of tick-borne pathogens in dogs and ticks from Nigeria. PLoS Negl Trop Dis 7:e2108

Levin ML, Killmaster LF, Zemtsova GE (2012) Domestic dogs (Canis familiaris) as reservoir hosts for Rickettsia conorii. Vector Borne Zoonotic Dis 12:28-33

Livanova NN, Fomenko NV, Akimov IA, Ivanov MJ, Tikunova NV, Armstrong R, Konyaev SV (2018) Dog survey in Russian veterinary hospitals: tick identification and molecular detection of tick-borne pathogens. Parasit Vectors Nov 14(1):591 11(

Mokhtar AS, Lim SF, Tay ST (2013) Molecular detection of Anaplasma platys and Babesia gibsoni in dogs in Malaysia. Trop Biomed 30:345-348

Mura A, Masala G, Tola S, Satta G, Fois F, Pirns P, Rolain J-M, Raoult D, Parola P (2008) First direct detection of rickettsial pathogens and a new rickettsia, 'Candidatus R. barbariae', in ticks from Sardinia, Italy. Clin Microbiol Infect 14(11):1028-1033

Sekeyova Z, Roux V, Raoult D (2001) Phylogeny of Rickettsia spp. inferred by comparing sequences of 'gene D', which encodes an intracytoplasmic protein. Int J Syst Evol Microbiol 51:1353-1360

Szabó MP, Mangold AJ, João CF, Bechara GH, Guglielmone AA (2005) Biological and DNA evidence of two dissimilar populations of the Rhipicephalus sanguineus tick group (Acari: Ixodidae) in South America. Vet Parasitol 130(1-2):131-140

Tamura K, Stecher G, Peterson D, Filipski A, Kumar S (2013) MEGA6: Molecular evolutionary genetics analysis version 6.0. Mol Biol Evol 30(12):2725-2729

Trotta M, Nicetto M, Fogliazza A, Montarsi F, Caldin M, Furlanello T, Solano-Gallego L (2012) Detection of Leishmania infantum, Babesia canis, and rickettsiae in ticks removed from dogs living in Italy. Ticks Tick Borne Dis 3(5-6):294-297

Vitale G, Mansuelo S, Rolain JM, Raoult D (2006) Rickettsia massiliae human isolation. Emerg Infect Dis 12(1):174

Waner T, Keysary A, Eremeeva ME, Din AB, Mumcuoglu KY, King R, Atiya-Nasaqi Y (2014) Rickettsia africae and Candidatus Rickettsia barbariae in ticks in Israel. Am J Trop Med Hyg 90(5):920-922

Wei QQ, Guo LP, Wang AD, Mu LM, Zhang K, Chen CF, Zhang WJ, Wang YZ (2015) The first detection of Rickettsia aeschlimannii. and Rickettsia massiliae in Rhipicephalus turanicus ticks, in northwest China. Parasit Vectors 8(1):1-4

Wood H, Artsob H (2012) Spotted fever group rickettsiae: a brief review and a Canadian perspective. Zoonoses Public Health 59(S2):65-79

Yabsley MJ, McKibben J, Macpherson CN, Cattan PF, Cherry NA, Hegarty BC, Breitschwerdt EB, O’Connor T, Chandrashekar R, Paterson T, Perea ML, Ball G, Friesen S, Goedde J, Henderson B, Sylvester W (2008) Prevalence of Ehrlichia canis, Anaplasma platys. Babesia canis vogeli, Hepatozoon canis, Bartonella vinsonii berkhoffi, and Rickettsia spp. in dogs from Grenada. Vet Parasitol 151:279-285

Yunik ME, Galloway TD, Lindsay LR (2015) Assessment of prevalence and distribution of spotted fever group rickettsiae in Manitoba, Canada, in the American Dog Tick. Dermacentor variabilis (Acari: Ixodidae). Vector Borne Zoonotic Dis 15(2):103-108

Zhao SS, Li HY, Yin XP, Liu ZQ, Chen CF, Wang YZ (2016) First detection of 'Candidatus R. barbariae', in the flea Vermipsylla alakurt, from north-western China. Parasit Vectors 9(1):325 
Zheng W, Liu M, Moumouni PF, Liu X, Efstratiou A, Liu Z, Efstratiou A, Liu Z, Liu Y, Tao H, Guo H, Wang G, Gao Y, Li Z, Ringo AE, Jirapattharasate C, Chen H, Xuan X (2017) First molecular detection of tick-borne pathogens in dogs from Jiangxi, China. J Vet Med Sci 79(2):248-254

\section{Affiliations}

Wurelihazi Hazihan ${ }^{1} \cdot$ Zhihui Dong $^{2}$ - Liping Guo ${ }^{3} \cdot$ Kadyken Rizabek ${ }^{4}$. Dzhunysov Askar ${ }^{4} \cdot K_{\text {Kulmanova Gulzhan }}^{4} \cdot$ Mahanov Kudaibergen $^{4}$. Akishev Nurlan Kenjebaevich ${ }^{4} \cdot$ Tolegen Talgat $^{4} \cdot$ Kenesbay Kairullayev $^{4}$. Yuanzhi Wang ${ }^{2}$

Wurelihazi Hazihan

wurelihazi@shu.edu.cn

Zhihui Dong

751453835@qq.com

Liping Guo

lipingguo229@126.com

Kadyken Rizabek

agajai@mail.r

Dzhunysov Askar

kayrat_ishan@mail.ru

Kulmanova Gulzhan

gulzhan_62@mail.ru

Mahanov Kudaibergen

makhanov_k@mail.ru

Akishev Nurlan Kenjebaevich

akishev_n@mail.ru

Tolegen Talgat

talgat_29-12-95@mail.ru

Kenesbay Kairullayev

kenes53@mail.ru

1 School of Animal Science and Technology, Shihezi University, Shihezi 832000, Xinjiang, China

2 School of Medicine, Shihezi University, Xinjiang Uygur Autonomous Region, Shihezi 832002, China

3 School of Medicine, Sun yat-sen university, Guangzhou 510080, China

4 Department of Food Engineering, Kazakh National Agrarian University, Almaty 050010, Kazakhstan 